\title{
Ultra High-Speed Transaxial Image Reconstruction of the Heart, Lungs, and Circulation via Numerical Approximation Methods and Optimized Processor Architecture
}

\author{
Barry K. Gilbert, ${ }^{*}$ Aloysius Chu, ${ }^{*}$ Daniel E. AtKins, $\dagger$ \\ EARl E. Swartzlander, Jr., $\ddagger$ AND ERIK L. RITMAN*
}

\begin{abstract}
* Biodynamics Research Unit, Department of Physiology and Biophysics, Mayo Foundation, Rochester, Minnesota 55901; † Department of Electrical and Computer Engineering, University of Michigan, Ann Arbor, Michigan 48109; and $\ddagger$ Electronic Systems Division, TRW Defense and Space Systems Group, Redondo Beach, California 90278
\end{abstract}

Received February 13, 1978

\begin{abstract}
A high temporal resolution scanning multiaxial tomography unit, the Dynamic Spatial Reconstructor (DSR), presently under development will be capable of recording multiangular Xray projection data of sufficient axial range to reconstruct a cylindrical volume consisting of up to 240 contiguous $1-\mathrm{mm}$ thick cross sections encompassing the intact thorax. At repetition rates of up to 60 sets of cross sections per second, the DSR will thus rccord projection data sufficient to reconstruct as many as 14400 cross-sectional images during each second of operation. Use of this system in a clinical setting will be dependent upon the development of software and hardware techniques for carrying out $\mathrm{X}$-ray reconstructions at the rate of hundreds of cross sections per second. A conceptual design, with several variations, is proposed for a special purpose hardware reconstruction processor capable of completing a single cross section reconstruction within 1 to $2 \mathrm{msec}$. In addition, it is suggested that the amount of computation required to execute the filtered back-projection algorithm may be decreased significantly by the utilization of approximation equations, formulated as recursions, for the generation of internal constants required by the algorithm. The effects on reconstructed image quality of several different approximation methods are investigated by reconstruction of density projections generated from a mathematically simulated model of the human thorax, assuming the same source-detector geometry and X-ray flux density as will be employed by the DSR. These studies have indicated that the prudent application of numerical approximations for the generation of internal constants will not cause significant degradation in reconstructed image quality and will in fact require substantially less auxiliary memory and computational capacity than required by direct execution of mathematically exact formulations of the reconstruction algorithm.
\end{abstract}

\section{INTRODUCTION}

Diagnostic radiology has been revolutionized by the advent of computerized Xray transaxial tomography, which allows the creation of cross-sectional images of any part of the body from the measured intensity of a beam of X-rays after 
penetration through the object under study. The spatial resolution of these images has improved steadily; currently available commercial scanners generate image matrices of up to $512 \times 512$ volume picture elements, ${ }^{1}$ or voxels. Advances in the design of X-ray source and detector systems for these machines have steadily decreased the duration required for recording the necessary $\mathrm{X}$-ray projection data from several minutes to 5 to $10 \mathrm{sec}$ for each cross section. The possibility that these recording durations may decrease even further is supported by a recently published description of a device which would record within $10 \mathrm{msec}$ the projection data necessary for a single cross section (2). Concurrent advances in the computer techniques by which the X-ray projection data is converted into cross-sectional images, initially by means of general purpose minicomputers, and more recently via special purpose hardware processors, have over the past 6 years decreased computation times from several minutes for an $80 \times 80$ voxel reconstruction to a few tens of seconds for the $512 \times 512$ images produced by the most recently announced commercial scanners (3).

\section{The Dynamic Spatial Reconstructor}

The commercially available single cross section scanners are, however, incapable of recording projection data with high temporal resolution and high spatial resolution in a direction orthogonal to the plane of reconstruction (i.e., the "axial" direction). Consequently, parallel cross sections generated by these machines do not provide accurate information concerning the three-dimensional anatomic shape and dynamic changes in the structural dimensions of rapidly moving organs such as the intact beating heart. Recognition of this limitation has led to the development of an advanced, high temporal resolution cylindrical scanning multiaxial tomography unit, the Dynamic Spatial Reconstructor (DSR). The DSR is designed to scan up to 240 $1-\mathrm{mm}$ thick cross sections within a duration of $11 \mathrm{msec}$, and to repeat this complete scan procedure at $\frac{1}{60}-\mathrm{sec}$ intervals, allowing the entire three-dimensional anatomic extent, as well as the dynamic changes in shape and dimensions, of moving structures such as the intact beating heart, lungs, and vascular system to be reconstructed (4-8).

Figure 1 depicts in schematic form the multiple X-ray source and detector assemblies of a proposed Dynamic Spatial Reconstructor system, consisting of a set of 28 independently controlled rotating-anode X-ray sources arranged around a semi-circle with a radius of $143 \mathrm{~cm}$, and a $30-\mathrm{cm}$ wide fluorescent screen bent around an opposing semicircle with a radius of $58 \mathrm{~cm}$. Twenty-eight sets of independently controlled image intensifiers and image isocon video cameras are arranged behind

\footnotetext{
'The term voxel, unlike the more familiar abbreviation, pixel (picture element), underscores the fact that each reconstructed image element represents a rectangular parallelepiped of tissue in a cross sectional slab of finite thickness $(I)$.
} 
the fluorescent screen to produce 28 video images of the $30-\times 30-\mathrm{cm}$ overlapping Roentgen projection images produced on the screen.

The entire assembly of $28 \mathrm{X}$-ray sources, curved fluorescent screen, and video camera chains will be mounted on a cylindrical aluminum gantry which will be rotated at a constant angular velocity of $90 \% / \mathrm{sec}$ about a horizontal axis which is colinear with the cephalocaudal axis of the subject. In normal operation, each X-ray source will be pulsed-on sequentially to irradiate the subject for a duration of 380 microsec, while simultaneously, its associated image intensifier and video camera are

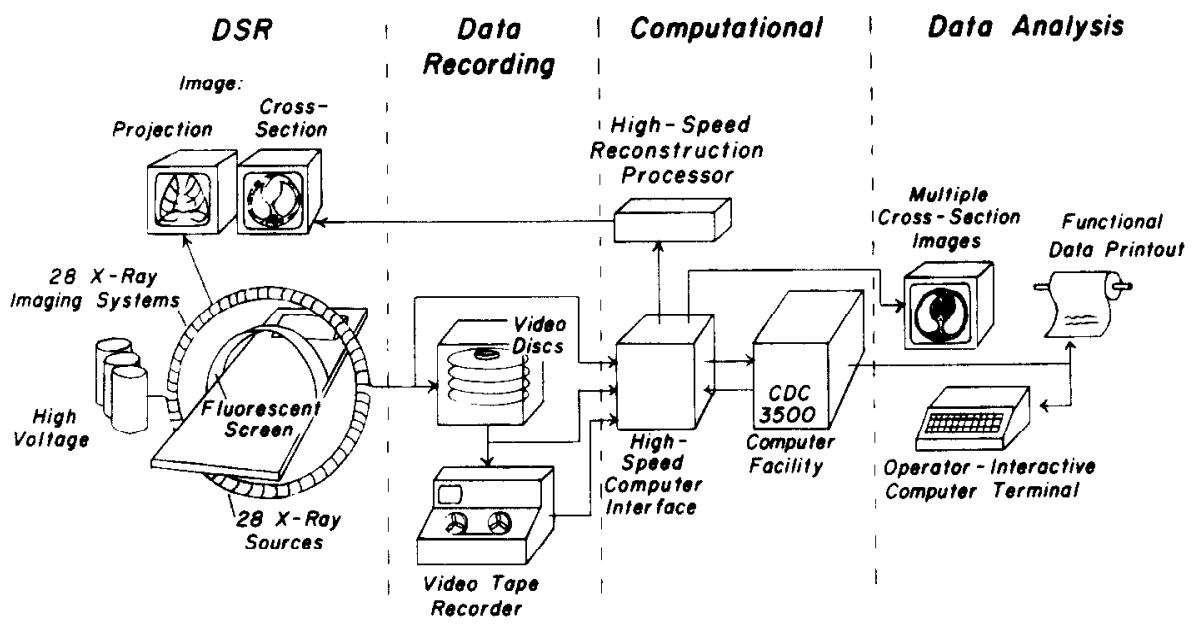

FIG. 1. Schematic of major components of Dynamic Spatial Reconstructor system. Roentgen video projection images from 28 video cameras radially mounted on the rotating gantry are multiplexed and stored on video discs. Projections necessary to reconstruct each cross section are replayed from disc and digitized by a high-speed digital data handling interface. Reconstructions are carried out by a general purpose computer (CDC 3500) or a hardwired digital reconstruction unit. (Reproduced with permission from (19).)

activated so as to record the image formed on the diametrically opposite portion of the curved fluorescent screen.

The number of angles of view incorporated into the reconstruction can be modified in the following manner. Although during rotation the gantry will move continuously around $360^{\circ}$, for purposes of system control, 720 discrete $0.5^{\circ}$ angular increments of the gantry position will serve as "firing stations" at which any one of the $28 \mathrm{X}$-ray tubes can be fired. The DSR scanner system is functionally highly flexible in that numerous variations of scanning configurations are available to permit quantitative assessment of the relative importance of transverse section thickness, image contrast, spatial and temporal resolution, and related computerized reconstruction algorithms and display techniques $(6,7,9)$. Specifically, spatial resolution of the reconstructions can be improved at the expense of temporal resolution by increasing the number of 
projections from 28 to 56 or 112 angles of view around approximately $180^{\circ}$ as the gantry rotates a full $360^{\circ}$ about its horizontal axis. The corresponding exposure times required for recording all the projections will increase from $11 \mathrm{msec}$ to 27.7 or $60.8 \mathrm{msec}$.

\section{ApPLiCATIONS OF THE DSR}

Proposed experimental protocols for diagnosis of, for example, the presence and extent of coronary artery disease and associated transmural distribution of myocardial perfusion (10) require the recording of approximately $15 \mathrm{sec}$ (i.e., 15-20 cardiac cycles) at the rate of $10 \mathrm{scans} / \mathrm{sec}$, in addition to at least 50 to 100 scans within a period of $1 \mathrm{sec}$ to delineate adequately the dynamic shape and dimensions of the myocardium. Consequently, up to 250 sets of projection data from each subject will be recorded throughout the duration of a complete study. On the assumption that reconstruction of only 60 of the available 240 cross sections from each scan will be undertaken, a processing load of approximately 14,400 127- $\times 127$-element cross sections is anticipated for each subject studied. If processing times of 1 to $10 \mathrm{sec}$ per cross section are eventually achieved with high-speed, general purpose computers executing optimized reconstruction programs or with special commercially available hardwired processors, computation of 14400 cross sections would require 4 to 40 hr. In addition to the resulting unsatisfactory delay between scan and diagnosis for any one patient, scanning of more than six patients per day would effectively be prevented, even though each scan procedure would involve approximately $\frac{1}{2} \mathrm{hr}$. Consequently, use of the DSR as a routine cost-effective clinical diagnostic technique for early detection and study of cardiovascular abnormalities and early lung cancer would be impossible $(4,7,8)$. It is anticipated that reconstruction times for each 127 $\times 127$-element cross section must be reduced to durations not exceeding 1 to 10 msec per image $(11-13)$, i.e., to at most a few minutes of computation for each scan procedure, if the DSR is to achieve its maximum investigative and clinical diagnostic potential.

\section{Computational Requirements of Reconstruction Algorithms}

Studies of the computational demands of the DSR indicate that no presently available general purpose computers operating unaided will be capable of fulfilling the data processing requirements of this device at reasonable cost. Thus, it will be necessary to utilize or develop special purpose arithmetic processors specifically optimized to execute efficient reconstruction algorithms.

One class of reconstruction algorithms which is currently quite appealing for execution by special purpose processors employs the filtered back-projection method (3). The first stage of this algorithm requires the filtering of each $\mathrm{X}$-ray projection, 
either by direct one-dimensional convolution in the spatial domain, or by means of the Fast Fourier Transform (FFT) or number-theoretic transforms (14) and direct vector multiplication of the data vector and filter function vector in the frequency domain (3). From the view of hardware implementation, the convolution and frequency domain filtration techniques each have both strengths and deficiencies. The design and fabrication of direct convolution hardware is straightforward; however, a greater number of arithmetic operations must be executed than required by any of the several versions of the FFT. The relative merits of the various filtration schemes are of major concern in view of the large numbers of projections which must be filtered to reconstruct the many thousands of cross sections.

The second portion of the algorithm is the back projection of the filtered projections into the image space to generate the halftone values of the image voxels. Explanations of the physical meaning of the back-projection algorithm are abundant (3) and will be described only briefly here. To back project a voxel, one sample, or ray sum, from each of the filtered projections is selected according to a prespecified selection algorithm; all ray sum values are then added together to create the halftone value of the voxel. Stated another way, since each ray sum represents the sum of the Roentgen densities of all voxels through which its associated X-ray traversed, back projection is the procedure by which the magnitude of each ray sum is partitioned among those voxels. In addition, if the individual rays in the planar X-ray beam diverge in their passage through the structure, the intensity of the unattenuated bearn decreases with increasing distance from the source. Hence, a weighting term must be incorporated into the calculation of the contribution of each ray sum to its associated image voxels as a function of the relative locations of the source and the voxel. The back projection equations for data collected with such a fan-shaped beam of X-rays are formulated so that the individual $\mathrm{X}$-ray which passed through each image voxel at a given angular position of the $\mathrm{X}$-ray source is identified, its corresponding ray sum is indexed from the array of all measured ray sums, and interpolation between measured ray sums is executed if a measured ray did not traverse the voxel in question. The appropriate weighting factor is then calculated and applied to the ray sum to produce the contribution to the voxel from that source position. The procedure is repeated for all angular positions of the $\mathrm{X}$-ray source until the voxel has been completely back projected, and then repeated for the remaining image voxels.

The equations for fan-beam back projection, assuming a curved X-ray detector strip, reproduced from (15), are

$$
\begin{gathered}
f(r, \phi) \approx \sum_{j=1}^{M} W_{j, r, \phi} W_{j, K(j, r, \phi)}, \\
K(j, r, \phi)=\tan ^{-1}\left\{\frac{r \cos \left(\beta_{j}-\phi\right)}{D+r \sin \left(\beta_{j}-\phi\right)}\right\},
\end{gathered}
$$




$$
\begin{aligned}
W_{j, r, \phi} & =\frac{\beta_{j+1}-\beta_{j-1}}{2 U^{2}}, \\
U & =\left[\left(r \cos \left(\beta_{j}-\phi\right)\right)^{2}+\left(D+r \sin \left(\beta_{j}-\phi\right)\right)^{2}\right]^{1 / 2}
\end{aligned}
$$

in which $f(r, \phi)$ is the magnitude of a back projected voxel at location $(r, \phi)$ (the sign of $\phi$ is positive for counterclockwise angular displacements measured from the positive $x$-axis), and the $Y_{j, K}$ represents the $K$ th ray sum from the $j$ th filtered projection; the subscripts on $Y$, i.e., $(j, K(j, r, \phi))$, represent the index of ray sum $Y$, or the ray sum index, referred to the image voxel at location $(r, \phi)$, from the $j$ th projection. Although the subscript $j$ is an independent variable, $K$ must be computed from Eq. [2]. The $W_{j, r, \phi}$ represents a multiplicative weight, applied to the $K$ th ray sum from the $j$ th filtered projection undergoing back projection into the image voxel at location $(r, \phi) . D$ represents the distance between the X-ray source and the center of the object; $\beta_{j}$ is the angle between the $y$-axis and a line connecting the $j$ th source position and the center of the object.

\section{Overview of Back Projection Implementations}

Two significantly different physical implementations of Eqs. [1]-[3], referred to as "projection-driven" and "voxel-driven" back projection, have been proposed. In the case of projection-driven back projection, commonly implemented in high-level languages for standard general purpose serial-instruction serial-data (SISD) computers, the $j$ th projection is filtered, its contribution to every voxel in the cross section is computed, the projection is discarded and replaced with the $(j+1)$ st projection, and the procedure is repeated. That is, the ray sums for each angle of view are back projected for all image voxels before proceeding to the next angle of view (projection). The linearity property of Eq. [1] allows the contribution of each projection to be computed independently and added to the running sums of contributions of all other projections. Sequential processing of projections conserves computer memory, since the data buffer allocated to the program need only be large enough to accommodate the $n^{2}$ image voxels of an $n \times n$ image and a single projection at any stage of the procedure. The ray sum contributions to each voxel are added to the accumulated contributions of previously processed projections by reading the contents of the memory location assigned to that voxel, adding the contribution from the projection currently undergoing back projection, and writing the updated summation back into the same location within the image memory. Back projection of $M$ projections to form an image by this procedure requires at least $(M-1) n^{2}$ memory read operations and $M n^{2}$ memory writc operations, i.c., ( $2 M-$ 1) $n^{2}$ memory references.

Voxel-driven back projection is defined here as a parallel processing approach in which all filtered projections must be available continuously throughout the entire 
duration of the image formation. For this procedure, all the projection ray sums, one from each angle of view, whose rays intersect a single image voxel are back projected before proceeding to the next voxel. Such an implementation is highly parallel because the contributing ray sums from all projections are indexed simultaneously and then summed simultaneously with the aid of one or more forms of nonsequential addition techniques, e.g., an adder tree. Each voxel is thus completely back projected in a single set of arithmetic operations and not readdressed thereafter.

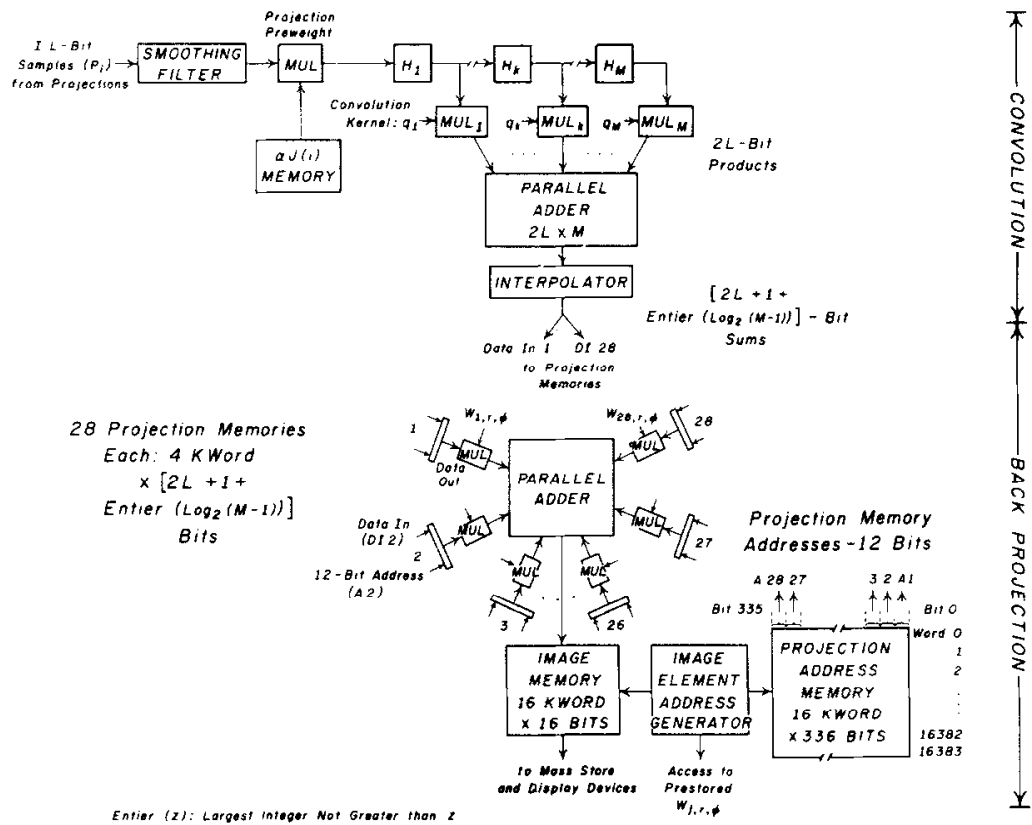

FIG. 2. Proposed design for hardwired parallel processing reconstruction computer executing fan beam convolution reconstruction. Filtration of each projection is via a direct digital convolution unit executing $\boldsymbol{M}$ multiplications and $\boldsymbol{M}-1$ additions simultaneously. Additional ray sums are interpolated into the filtered projections, which are then stored in individual projection memories. Each voxel is completely back projected, with contributions from all 28 projections simultaneously indexed, separately weighted, and summed in parallel via an adder tree.

Figure 2 is a schematic diagram of a hardware processor executing direct convolution filtration and voxel-driven back projection. In this example it is assumed, in agreement with the source-detector configuration of the DSR, that 28 projections will be back projected simultaneously. As the elements of each filtered projection emerge from the convolution processor, additional ray sums are incorporated into the projection by a hardware unit executing multiple stages of linear interpolations on the filtered ray sums taken pairwise. At the first stage of interpolation, a single value is computed midway between each pair of measured ray sums; in the second stage, two additional midpoints are calculated between the original pair of ray sums 
and the midpoint computed in the first stage. This procedure may be repeated through one or more additional stages as required, with $1,3,7,15$, or more computed ray sums included between each pair of measured ray sums when the interpolation has been completed. Interpolation may be executed in this manner with a binary adder tree only, obviating the requirement for execution of multiplication or division operations. Incorporation of interpolated ray sums into the filtered projections aids materially in the execution of the subsequent back projection, as described in (15). Execution of all interpolations unconditionally at a single strategically chosen location in the data flow pathway encourages the use of very efficient pipelined interpolation hardware; thereafter, during the back projection, only nearest-neighbor indexing of actual or "preinterpolated" ray sums is employed. Unlike the execution of linear interpolation by a computer program for an SISD computer, the procedure described here can be "buried" in the transmission of the filtered projections into high-speed storage buffers.

The filtered projections, in this example interpolated to a maximum of 4096 ray sums, are then stored in a group of "projection memories" prior to the onset of back projection. Each voxel in the reconstructed image is formed by summing one contribution from each of the 28 projection memories. Each projection memory contribution is created by reading the contents of one data word location, multiplying this value by the appropriate $W_{j, r, \phi^{*}}$ and applying the weighted data value to the inputs of a high-speed multiple operand parallel adder which forms the required image voxel density sum. For each voxel, a unique set of 28 addresses simultaneously specifies (i.e., indexes) the requisite ray sums within all of the 28 projection memories; for the $127 \times 127$ cross-sectional reconstruction there are 16129 unique sets of 28 12-bit addresses (to access any of the 4096 possible ray sums within a projection), which may be precalculated from Eq. [2] and prestored in a "projection address memory." As diagrammed in Fig. 2, reading of a single 336-bit word in the projection address memory results indirectly in the reading of the necessary ray sum from each of the projection memories to generate one image voxel. With the availability of a multiple operand adder (16) allowing the simultaneous summation of many ray sum contributions, each voxel would be generated within a single operating cycle of the processor; in addition, no read operations and only $n^{2}$ write operations into the image memory are required. Hence, with reference to fixed memory cycle times, back projection executed in parallel is potentially $(2 M-1)$ times faster than its sequential counterpart; because the parallel processing back projection unit may be fabricated from a very few hardware subunits of different design, each replicated a number of times, the cost in additional engineering design to achieve this increase in throughput is much less than $(2 M \ldots$ 1) times greater. Because of its inability to perform the same operations on multiple operands simultaneously, an SISD computer can neither mechanize nor exploit the computational parallelism inherent in the voxel-driven back projection algorithm.

In view of the reliance of the architecture of Fig. 2 upon reprogrammable memory 
to initiate and control all phases of algorithm execution, the design is referred to as a "memory-driven" processor. This mechanization possesses a substantial degree of flexibility, resulting from the ability of the user to redefine the step-by-step operation of the processor by modifying the contents of the projection and projection address memories. For example, a processor designed in accord with Fig. 2 can reconstruct nontransverse sections, or an entire transverse section with varying spatial resolution in different subregions of the cross section. When applied to the DSR, an additional capability of this implementation is of particular value. As originally proposed by Herman et al. (15), the fan beam convolution algorithm requires a set of projections equispaced about $360^{\circ}$ of the object to be reconstructed. Recently, Herman and Naparstek (17) have suggested a modification of the fan beam algorithm to permit reconstructions from projections equispaced over an angular range of $180^{\circ}$ plus the divergence angle $\alpha$ of the X-ray beam. This form of the algorithm is well suited to the source-detector geometry and rotational constraints of the DSR gantry which, because of its low rate of angular displacement, would exhibit reduced temporal resolution, from a scan time of $11 \mathrm{msec}$ for 28 views to about $4 \mathrm{sec}$ if required to record data from about the entire circumference of the object. The HermanNaparstek modification of the fan beam convolution algorithm requires that selected ray sums from projections recorded near $0^{\circ}$ and $(180+\alpha)^{\circ}$ angular displacement of the X-ray sources not be back projected at all, i.e., are to be assumed to have zero magnitude. Since the $j-K$ indices of these zero-magnitude ray sums are easily calculable, the voxel-driven back projector can directly exploit this modified algorithm by defining as identically zero the contents of one address location in each projection memory; the projection address memory is loaded with the addresses of these null locations for ray sums to be ignored during back projection, thereby directly satisfying the Herman-Naparstek modification. Null contributions of individual ray sums require no additional indexing operations and do not contribute to truncation or overflow error in a special purpose processor. The algorithm simulation studies described below employed the full $360^{\circ}$ version of the fan beam convolution, which provides a more stringent test of the numerical stability of the reconstruction procedure than does the Herman-Naparstek modification.

The feasibility of achieving reconstruction speeds which are sufficient for the large amounts of data generated by the Dynamic Spatial Reconstructor has been assessed through an examination of the computational loads generated by each portion of the reconstruction algorithm and associated preprocessing operations. Throughput constraints arising in the filtration of the individual projections have been addressed elsewhere (11-13). A high-speed implementation of Eqs. [1]-[3] is sensitive to the relative computational costs of the three major portions of the back projection algorithm, i.e., the indexing via Eq. [2] of the ray sums required to back project a given voxel, the computation via Eq. [3] of the magnitudes of the back projection weights, the $W_{j, r, \phi}$, and the multiple operand addition of these ray sums to form the completely back projected voxel. 
Feasible implementations of the high-speed multiple operand adder implied by the summation operation of Eq. [1] have been described in $(12,16)$. The kernel of Eq. [1] requires a multiplicative weighting of the individual ray sums from the filtered projections by the $W_{j, r, \phi}$ with a concomitant nontrivial but manageable increase in system complexity or execution time.

\section{Optimization of the Back Projection Computations}

At least three mechanizations of Eqs. [2] and [3| for execution by general purpose or special purpose processors are apparent. Equations [2] and [3] may be evaluated directly for each voxel and each projection every time a reconstruction is made. Alternately, for a given source-object-detector geometry, and irrespective of X-ray scattering (8), the ray sum indices and $W_{j, r, \phi}$ need only be computed once via Eqs. [2]-[3] and then stored, and indexed by voxel number thereafter. However, as is illustrated by an example, either the direct numerical evaluation in real time of the $W_{j, r, \phi}$, or their precalculation and storage, is a significant portion of the entire back projection which demands high-speed computational hardware capable of manipulating trigonometric functions or additional high-speed (i.e., core or solidstate) memory to store the precalculated $W_{i, r . \phi}$. Equation [3] demonstrates that the magnitudes of the $W_{j, r, \phi}$ vary with image voxel position and with the angular index of each projection. An image of $n^{2}$ voxels reconstructed from $M$ projections requires $M n^{2}$ evaluations of $W_{j, r . \Phi}$; for an image of $127^{2}$ voxels reconstructed from 100 projections, real-time computational capability or storage would be required for 1600000 values of $W_{j, r, \phi}$.

A compromise between the two approaches described above can reduce the amount of repetitive computation below that required for direct evaluation of Eqs. [2] and [3] and also decrease storage requirements to well below that of the look-up approach. The exactly calculated values of $K(j, r, \phi)$ and/or $W_{j, r, \phi}$ are employed to develop a set of approximation equations in which the approximate values, hereafter denoted as $K(j, r, \phi)$ and $\hat{W}_{j, r, \phi}$, are dependent variables and the image voxel coordinates the independent variables. The form of these equations is selected to yield satisfactorily close approximations to the exact values with a minimum of computation and a minimum number of prestored constants.

Figure 3 depicts reconstructions of a mathematically generated test object with approximate dimensions of 28 by $35 \mathrm{~cm}$ which simulates a single cross section of a human thorax, including lung parenchyma, sternum, ribs, vertebrae, and an elliptical structure which represents the radiopacity of a chamber of the heart filled with a blood-X-ray contrast medium mixture containing $7 \mathrm{mg}$ iodine $/ \mathrm{ml}$. The linear absorption coefficients of these substructures were assigned on the basis of actual physical measurements $(8)$. The ventricular chamber of this "heart" is simulated by a 7.6- $\mathrm{cm}$ circular disc, the smooth edge of which is interrupted at $90^{\circ}$ angular intervals by sawtooth serrations (equilateral triangles) of dimension $8,4,2$, and $1 \mathrm{~mm}$, to 
simulate the physical size of the papillary muscles and trabeculae carnae. The X-ray contrast of the disc is assumed to be that which would result from the peripheral venous injection of $100 \mathrm{ml}$ of $69 \%$ renovist, resulting in a $2 \%$ concentration of contrast material in the left ventricular chamber, and an increase of $20 \%$ in the linear absorption coefficient of the blood in the chamber. Voxel size in these reconstructions is $3 \times 3 \times 4 \mathrm{~mm}$; hence, only the 8- $\mathrm{mm}$ triangular serrations can be resolved.

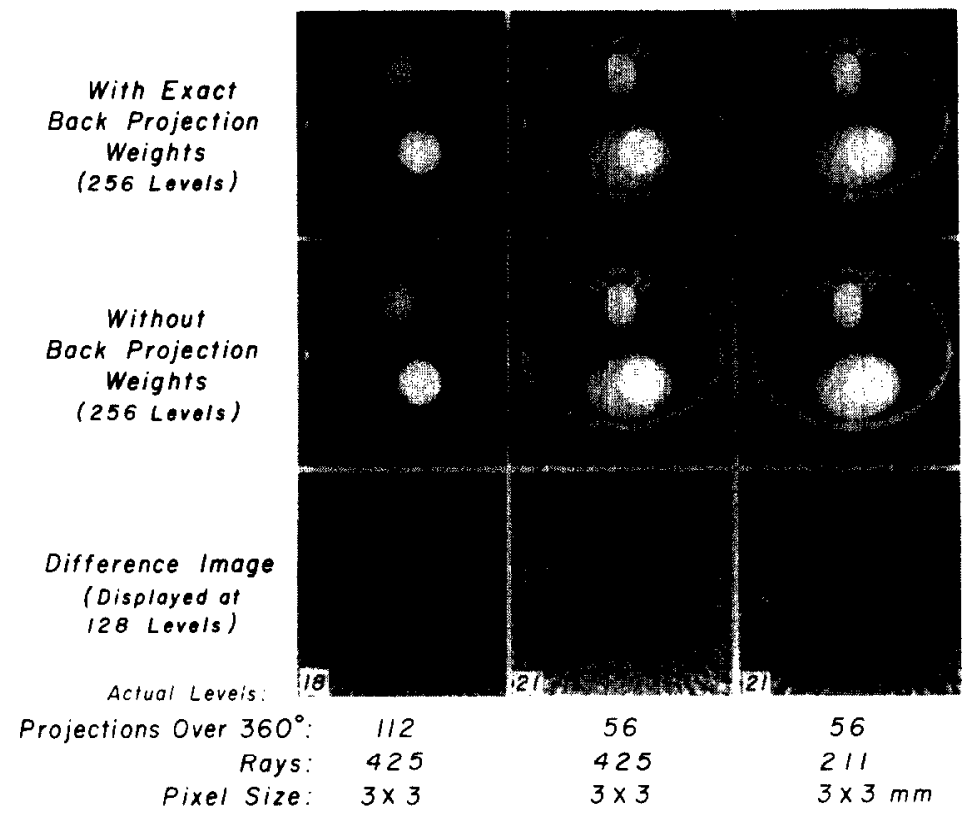

Fig. 3. Degradation of reconstructed image quality caused by deleting contribution of $W_{j, r, \phi}$, the back-projection weighting factor. Upper row of $127 \times 127$ images reconstructed via Eqs. [1]-[3], i.e., using the exactly computed values of $W_{j, r, \phi}$. Middle row of images reconstructed via Eqs. [1] and [2] from same projection data, but with all $W_{j, r, \theta}$ set equal. The structure apparent in difference images indicates reconstruction error attributable to neglect of $W_{j, r, \theta}$ factors. "Actual levels" are peak-to-peak halftone range as represented by brightest and darkest voxels in difference images prior to gray scale enhancement for display.

The projections which served as input data to the reconstructions were mathematically generated (18) under the assumption of a fan-beam of $70-\mathrm{keV}$ monoenergetic X-rays with divergency angle of $15^{\circ}$, with a DSR source-to-detector distance of $201 \mathrm{~cm}$, and a center-of-object-to-detector distance of $58 \mathrm{~cm}$. The incident X-ray beam was assumed to contain a mean of 200000 photons per ray, the exact number of photons in each ray fluctuating according to a Poisson statistical distribution. For this figure three sets of reconstructions were carried out in 48-bit floating point arithmetic on a Control Data Corporation CDC 3500 computer using varying numbers of projections, in every case equispaced around $360^{\circ}$, and different 
numbers of ray sums in each projection. The reconstructions in the upper row of Fig. 3 were carried out using the $W_{j, r, \phi}$ as specified by Eq. [3]; the resulting cross sections were displayed on and photographed from a RAMTEK video graphics device (9) at 256 halftone levels ( 8 bits). In the middle row of images, these reconstructions were repeated but with all $W_{j, r, \phi}$ set equal, and displayed on the RAMTEK in the same manner. Thereafter, the halftone values of the reconstructions in the middle row of

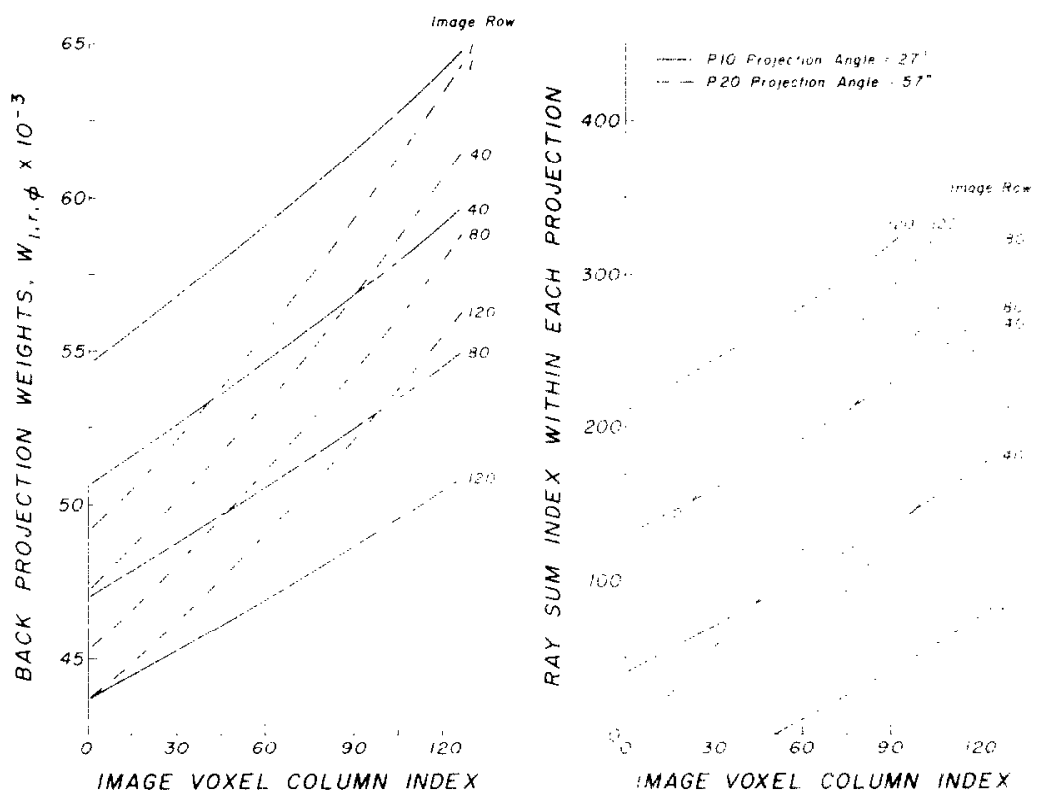

FIG. 4. Justification for use of polynomial approximations to generate $W_{j, r . \forall}$ (left panel) and ray sum indices $K(j, r, \phi)$ (right panel) for fan beam convolution reconstruction algorithm. Back projection of image is to be row by row; if parameterized by image row and by projection index $j$, values of $W_{i, r . A}$ and $K(j, r, \phi)$ appear as families of nearly linear functions of the voxel column index.

each column were subtracted voxel by voxel from those of the upper row; the resulting difference images, with their gray scale ranges linearly expanded for display purposes to 128 halftone levels ( 7 bits), appear in the lowermost row. In addition, the numbers inset on each lowermost panel specify the actual maximum halftone range, or "peak-to-peak" difference, between the darkest and brightest voxel in each difference image prior to the halftone expansion. To assure ease of comparison between the various reconstructions, none of the images of Fig. 3 (or of Figs. 5-9) was subjected to noise reduction or image enhancement techniques at any stage of preparation.

The difference images in Fig. 3 indicate the magnitude of the reconstruction error resulting from neglecting the $W_{j, r, \phi}$. Similar results have been obtained with 
projections equispaced around $180^{\circ}$ of the object, and for reconstructions of subsections of the thorax in which each voxel of the reconstructed image represented only a $1.5 \times 1.5-\mathrm{mm}$ area or less. These results are defined as the limiting case on the degradation caused by disregarding or approximating the $W_{j, r, \phi}$ assuming the geometry, flux density, and subject material of the DSR; the computational cost of calculating in real-time or prestoring the exact values of these constants can thus be

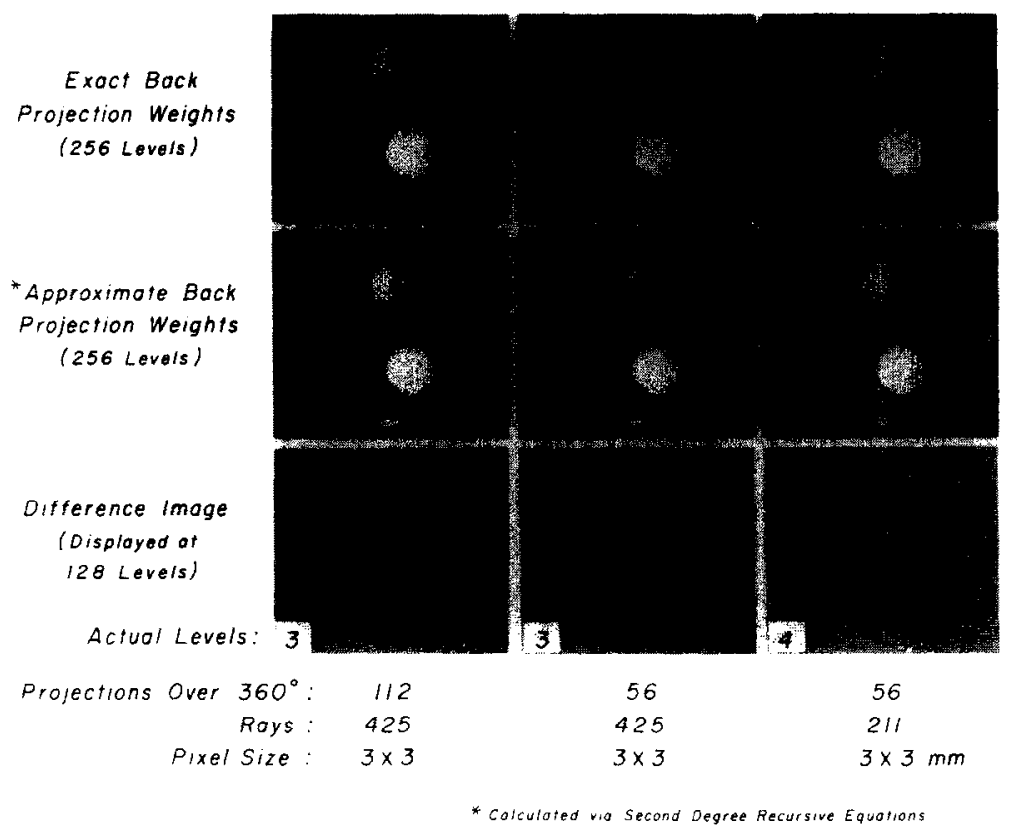

Fig. 5. Reconstructions of mathematically simulated human thorax employing projection data of Fig. 3 with exact $W_{j, r, \theta}$ from Eq. [3] (upper three images), and approximate values $\hat{W}_{j, y . x}$ computed via second-degree recursions (middle three images). "Actual levels" are defined as in Fig. 3 legend.

balanced against the image degradation resulting from alternate computational methods for these weights.

Although the back projection unit of Fig. 2 can generate the image elements in any desired sequence, an orderly column-by-column or row-by-row generation of the voxels is computationally advantageous. The left panel of Fig. 4 plots the magnitudes of the $W_{j, r, \phi}$ under the assumption that the voxels are back projected row by row. The ordinate displays the magnitude of the $W_{j, r, \phi}$; the abcissa represents the voxel column index within its row (vnxel zero is the leftmost of its row). For the sourceobject-detector geometry of the DSR, the left panel of Fig. 4 demonstrates that the magnitudes of the $W_{j, r, \phi}$ computed from Eq. [3] vary as a nearly linear function of column index for a given row in the reconstructed image. Consecutive approximated 
values $\hat{W}_{j, r, \phi}$, referred to the rows of the reconstructed image array, may thus be generated by polynomials whose constants are selected by means of a predetermined error criterion to give the best approximation to the $W_{j, r, \infty}$ on a row-by-row basis.

Initial implementations of this technique employed a quadratic form chosen to minimize the mean-squared difference between $W_{j, r, \phi}$ and $\hat{W}_{j, r, \phi}$ for all voxel column indices within each row of the reconstructed image, requiring storage of threc initial constants per row and for each projection. Thus, precalculation and storage of

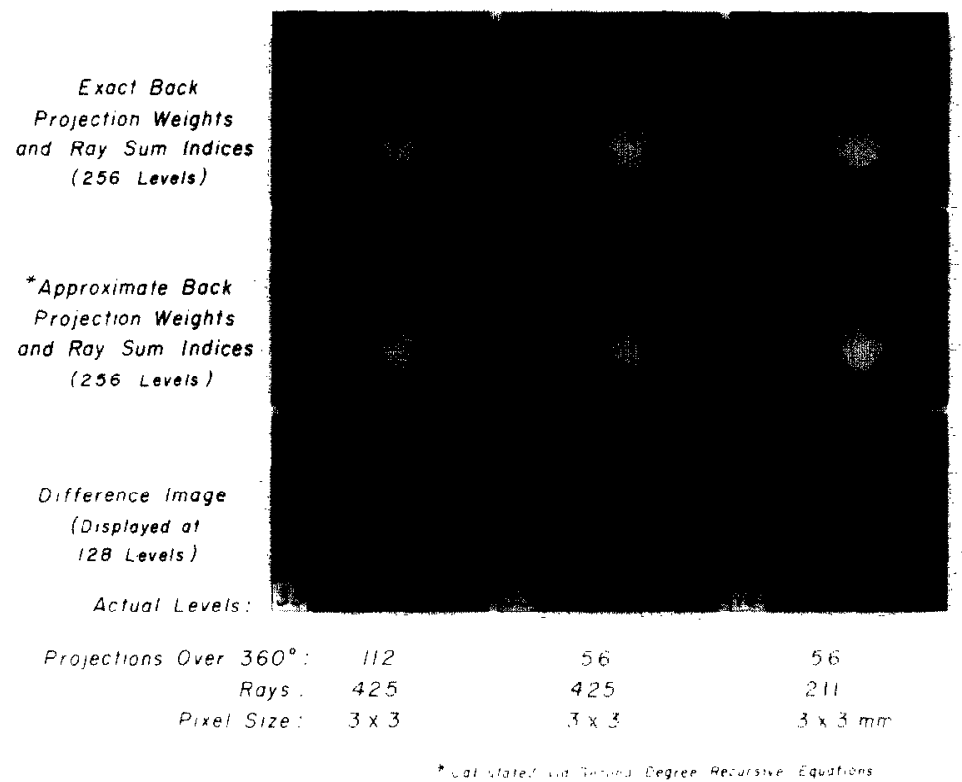

FIG. 6. Reconstructions of mathematically simulated human thorax employing second-degree recursions to generate both the $\hat{W}_{j, y, x}$ and ray sum indices for all image elements. The structuring in difference images and peak-to-peak error of 8 to $14 \%$ indicates substantial mismatch between the exactly reconstructed images (upper row) and images generated with approximations (middle row). Comparison with Fig. 5 indicates that calculation of ray sum indices requires greater accuracy than for $\hat{W}_{j, y, x}$

$38100(3 \times 127 \times 100)$ constants was sufficicnt to back project a $127 \times 127$ image from 100 projections. Direct evaluation of the quadratic requires the execution of three multiplications and two additions, or, applying Horner's rule, two multiplications and two additions. However, the quadratic may be reformulated into a second-degree interpolation expression, following Newton's forward difference formula, and then rearranged into a set of recursions of the form:

$$
\left.\begin{array}{rl}
\hat{W}_{j, y, x+1} & =\hat{W}_{j, y, x}+D 1_{j, y, x} \\
D 1_{j, y, x+1} & =D 1_{j, y, x}+D 2_{j, y}
\end{array}\right\} j=1,2, \ldots, M, y=1,2, \ldots, 127, x=2,3, \ldots, 127
$$


in which the change in subscript variables for the $\hat{W}$ is introduced to emphasize that the $\hat{W}$ are generated, with regard to an image matrix in Cartesian coordinates, sequentially across all voxels in each row of the image. $\hat{W}_{j, y, 1}, D 1_{j, y, 1}$, and $D 2_{j, y}$ are selected to minimize the mean-squared difference between $W_{j, y, x}$ and $\hat{W}_{j, y, x}$ over the range $2 \leqslant x \leqslant 127 ; D 2_{j . y}$ is a constant. Each evaluation of Eq. [4] requires only two add-accumulate operations and no multiplications. Using Eq. [4] and the same projection data employed for Fig. 3, the middle rows of reconstructions were repeated (Fig. 5). The results of Figs. 3 and 5, particularly the peak-to-peak differences between the exact reconstructions, those in which the $W_{j, r, \phi}$ were neglected, and those reconstructed with Eq. [4] suggest that the penalty for achieving a marked decrease in processing costs by the use of second-degree approximations is only a minimal amount of image degradation. Execution times of the fan beam filtered back projection programs employing Eq. [4] decreased by $25 \%$ in comparison to the durations required to complete the reconstructions via direct evaluation of Eq. [3].

The use of approximations was investigated as a mechanism for indexing the requisite ray sums for back projection into each image voxel. The rightmost panel of Fig. 4 demonstrates the feasibility of this proposal since the $K(j, r, \phi)$ vary in a nearly linear manner with voxel column index within each row of the image. The projection data of Fig. 3 were reconstructed using second-degree approximations of the form of Eq. [4] to generate appropriate values $\hat{R}(j, y, x)$; the subscripts on $\hat{K}$ have been modified to emphasize that the approximated values $\hat{K}$ are generated via recursion with regard to a Cartesian coordinate system embedded in the reconstructed image. Figure 6 depicts reconstructions carried out with second-degree approximation of both the $W_{j, y, x}$ and the $K(j, y, x)$. The large peak-to-peak values and the physical appearance of the difference images indicate that use of lower-degree approximations results in significant inaccuracies in the reconstructed images, particularly at contrast boundaries where errors in the selection of ray sums cause the apparent boundaries to be displaced slightly from their true locations. Approximation schemes for generation of the $R(j, y, x)$ thus appear to require greater accuracy than those for the $\hat{W}_{j, y, x}$. Of particular value would be an approximation technique of sufficient accuracy to allow both the $\hat{W}_{j, y, x}$ and the $\hat{K}(j, y, x)$ to be computed easily but without causing major degradation of the reconstructed images.

Improvements in the generation of the $\hat{R}(j, y, x)$ and $\hat{W}_{j, y, x}$ may be achieved at greater processing cost by use of higher-degree approximations. Third-degree Newton's forward difference formulas were written as recursions of the form:

$$
\begin{aligned}
& \hat{W}_{j, y, x+1}=\hat{W}_{j, y, x}+D 1_{j, y, x}, \\
& D 1_{j, y, x+1}=D 1_{j, y, x}+D 2_{j, y, x}, \\
& D 2_{j, y, x+1}=D 2_{j, y, x}+D 3_{j, y}, \quad j=1,2, \ldots, M, \quad y=1,2, \ldots, 127,
\end{aligned}
$$

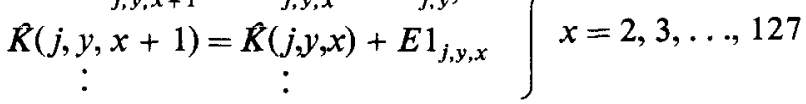


with the $\hat{W}_{j, y, 1}, \hat{K}(j, y, 1), D 1_{j, y, 1}, \ldots E 3_{j, y}$ chosen to minimize the mean-squared differences between the $W_{j, y, x}$ and the $\hat{W}_{j, y, x}$, and between the $K(j, y, x)$ and $\hat{K}(j, y, x)$ throughout the range $2 \leqslant x \leqslant 127 . D 3_{j, y}$ and $E 3_{j, y}$ are constants. Equation [5] was then used to reconstruct the projection data of Fig. 3; the results of these reconstructions are depicted in Fig. 7. The absence of identifiable structure in

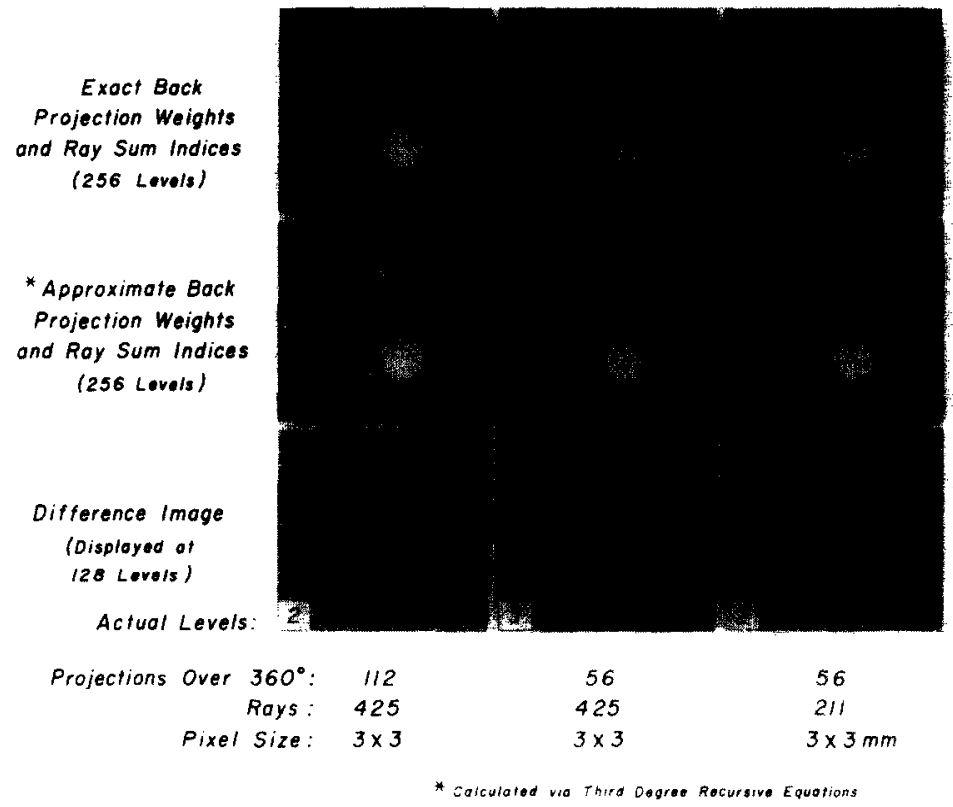

FIG. 7. Reconstructions of mathematically simulated human thorax using projection data of Fig. 3 with third-degree recursions to generate the $\hat{W}_{j, y, x}$ and ray sum indices for each image element (middle row). Upper row of $127 \times 127$ images were reconstructed exactly using Eq. [1]-[3]. Peak-to-peak error as demonstrated by difference images is approximately $1 \%$. Some structuring of difference images is visible.

the difference images, combined with a peak-to-peak error of only three halftone levels, i.e., approximately $1 \%$, indicates that the third-degree approximations result in quite accurate reconstructions requiring only three add-accumulate operations for each generated constant. The slight increase in computational cost of the thirddegree approximation method appears justifiable in view of the ability to generate recursively both the $\hat{W}_{j, y, x}$ and the $R(j, y, x)$ while maintaining reconstructed image quality nearly identical to that obtained with direct evaluation of Eqs. [1]-[3]. 
Fourth-degree recursions of the form:

$$
\left.\begin{array}{rl}
\hat{W}_{j, y, x+1} & =\hat{W}_{j, y, x}+D 1_{j, y, x}, \\
D 1_{j, y, x+1} & =D 1_{j, y, x}+D 2_{j, y, x}, \\
D 2_{j, y, x+1} & =D 2_{j, y, x}+D 3_{j, y, x}, \\
D 3_{j, y, x+1} & =D 3_{j, y, x}+D 4_{j, y}, \\
\hat{K}(j, y, x+1) & =\hat{K}(j, y, x)+E 1_{j, y, x}, \\
\vdots & \vdots
\end{array}\right\} \begin{aligned}
& j=1,2, \ldots, M, \quad y=1,2, \ldots, 127, \\
& x=2,3, \ldots, 127
\end{aligned}
$$

in which the $\hat{W}_{j, y, 1}, D 1_{j, y, 1}, \ldots, E 4_{j, y}$ were chosen to minimize the mean-squared differences between the exactly calculated $W_{j, y, x}$ and $K(j, y, x)$ and their approximated versions, were also employed to reconstruct the projection data of Fig. 3. The results of these studies, depicted in Fig. 8, indicate that reconstructions employing Eq. [6] are even more like the images generated exactly from Eq. [1]-[3] than those obtained via third-degree approximations; for example, peak-to-peak error decreased to less than $1 \%$. Equation [6] requires four add-accumulate operations to generate each constant, an additional computational cost which may be justifiable in certain cases, e.g., when computer postprocessing of the images is contemplated.

To decrease further the amount of storage necessary for the parameters of Eqs. [4], [5], or [6], an attempt was made to employ Eq. [5] to generate upon demand from a set of "super constants" the initial conditions, that is, the initial $D$ and $E$ values, required by the approximation method itself (i.e., Eq. [5]) for each row of the image and for each projection. As will be substantiated below, a marked decrease in the cost of processor design may be realized by use of such a two-stage approximation technique, in comparison to the single-stage approximations just described. To test the feasibility of such a mechanization, the projection data of Fig. 3 were employed to reconstruct a set of cross sections, using these "super constants," to generate the $\hat{K}(j, y, x)$ and $\hat{W}_{j, y, x}$ (Fig. 9). The additional degradation in image quality in comparison to Fig. 7 is negligible. However, attempts to use the seconddegree recursions of Eq. [4] to generate row-by-row initial conditions for a second application of Eq. [4] demonstrated reconstructed images with large halftone inaccuracies in a voxel-by-voxel comparison with the exact reconstructions generated via Eqs. [1]-[3].

Because approximation techniques conserve the size of storage required for precalculated constants, they are ideal for hardware implementation whenever a great many transaxial reconstructions must be executed with maintenance of a very high throughput rate, e.g., the DSR. Figure 10 depicts an alternate architecture to that of Fig. 2, which exploits the recursive generation of these constants. Although in accord with the configuration of the DSR, it was assumed for this figure that 28 
projections will be processed simultaneously, the design generalizes directly to any desired number of projection hardware subunits. With each projection memory is associated a recursive processor which generates $\hat{K}(j, y, x)$ and a second independent unit which computes the requisite values of $\hat{W}_{j, y, x}$. The weighted ray sums generated by the 28 identical projection memory units, each represented by the contents of the dashed box, are summed by means of an adder tree to form each reconstructed

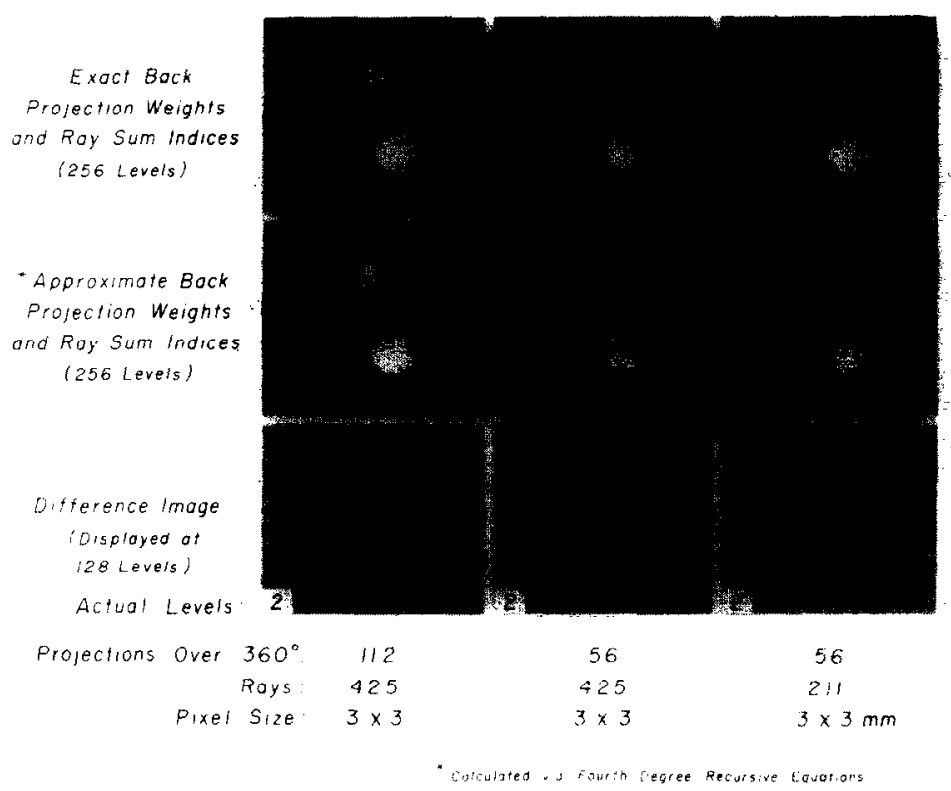

FIG. 8. Projection data of Fig. 3 reconstructed with the aid of fourth-degree recursions to generate the $\hat{W}_{j, y, x}$ and ray sum indices. Peak-to-peak error is less than $1 \%$, with absence of structuring in difference images. Fourth-degree recursions are the most computationally costly but accurate of approximation methods investigated.

voxel. Implementation of this design using readily available digital components will permit generation of the $\hat{K}(j, y, x)$ and $\hat{W}_{j, y, x}$, selection of the required ray sum contributions, and the presentation of all the weighted ray sums to the adder tree every 100 to $150 \mathrm{nsec}$; even higher throughput can be achieved for a slightly greater hardware complexity.

The major advantage of the architecture of Fig. 10 is that a minimal storage capacity is required for precalculated constants, which is of particular significance in the processing environment of the Dynamic Spatial Reconstructor. In the high temporal resolution mode of operation, in which 28 projections are recorded within 
$11 \mathrm{msec}$, using a single stage of Eq. [5] to generate both the $\hat{K}(j, y, x)$ and the $\hat{W}_{j, y, x}$ on a row-by-row basis, only $28 \times 10^{3}$ constants would require precalculation and storage, rather than $9 \times 10^{5}$ exactly computed $K(j, r, \phi)$ and $W_{j, r, \phi}$ values, i.e., a reduction by a factor of 32 in the number of constants. If a two-stage recursion using Eq. [5] is employed, only 896 constants would be precomputed and stored, a further reduction by a factor of 32 for a total reduction by a factor of 1024 compared to the

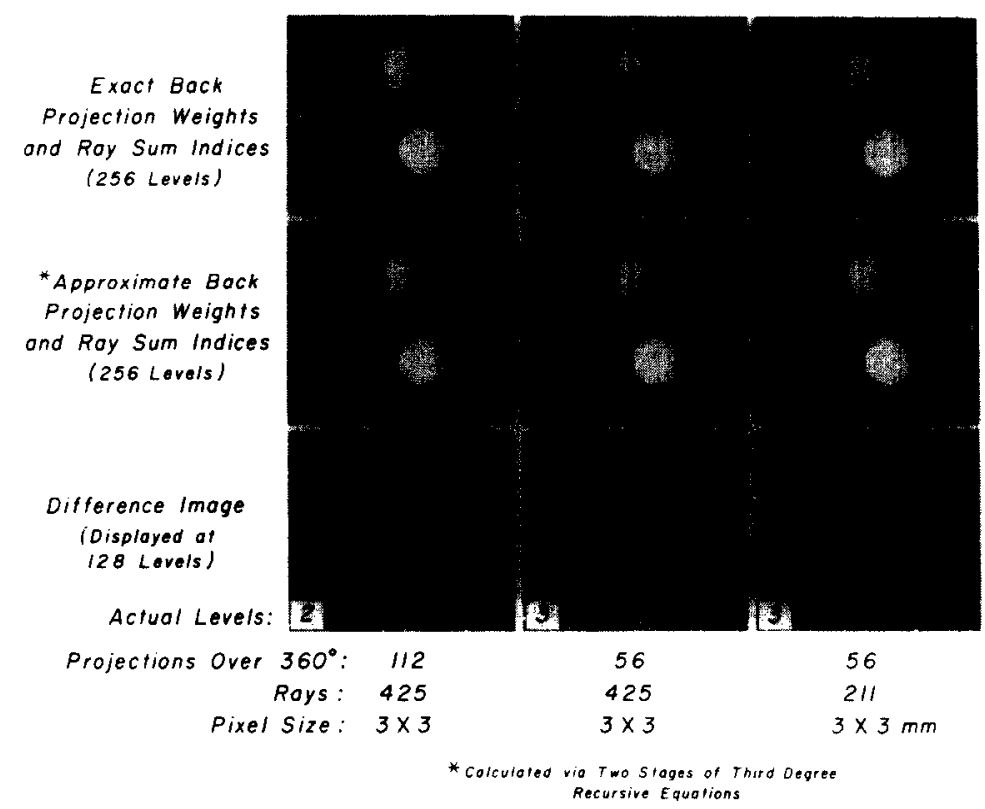

Fig. 9. Reconstructions of the projection data of Fig. 3, with $\hat{W}_{j, y, x}$ and ray sum indices computed with third-degree recursions, executed in two successive stages. First, Eq. [5] was employed to generate from one set of "super constants" the $D$ and $E$ values required for a second stage of evaluation of Eq. [5], during which the values of $\hat{W}_{j, y, x}$ and ray sum indices were actually computed. The number of constants requiring storage is reduced by a factor of 32 compared to a single stage of evaluation of Eq. [5]. Comparison with Fig. 7 indicates no significant additional degradation of reconstructed image quality in comparison to a single stage of evaluation of Eq. [5].

original approach. The saving is even greater for the high spatial resolution mode of operation, in which four (or more) sets of 28 views of the object will be recorded at $16 \mathrm{msec}$ intervals, i.e., the recording of 112 projections within approximately 61 msec. With 720 possible "firing stations" at $0.5^{\circ}$ intervals around the circumference of the gantry travel, 4 (or more) sets of back projections of 28 projections each must be computed from 4 (or more) of 720 possible X-ray tube firing positions with respect to the reconstruction coordinate system. If precomputation of the exact values of these constants via Eqs. 2 and 3 is employed, storage of $2.3 \times 10^{7} K(j, r, \phi)$ 
and $W_{j, r, \phi}$ will be necessary; conversely, the generation of these values as needed in real time using a single-stage recursion of the form of Eq. [5] would require storage of $7.3 \times 10^{5}$ constants, i.e., a factor of 32 reduction. If a two-stage recursion of the same form is employed, only $23 \times 10^{3}$ constants need be precalculated, with concomitant conservation of storage by a factor of 1024 from the original requirement to a presently feasible level.

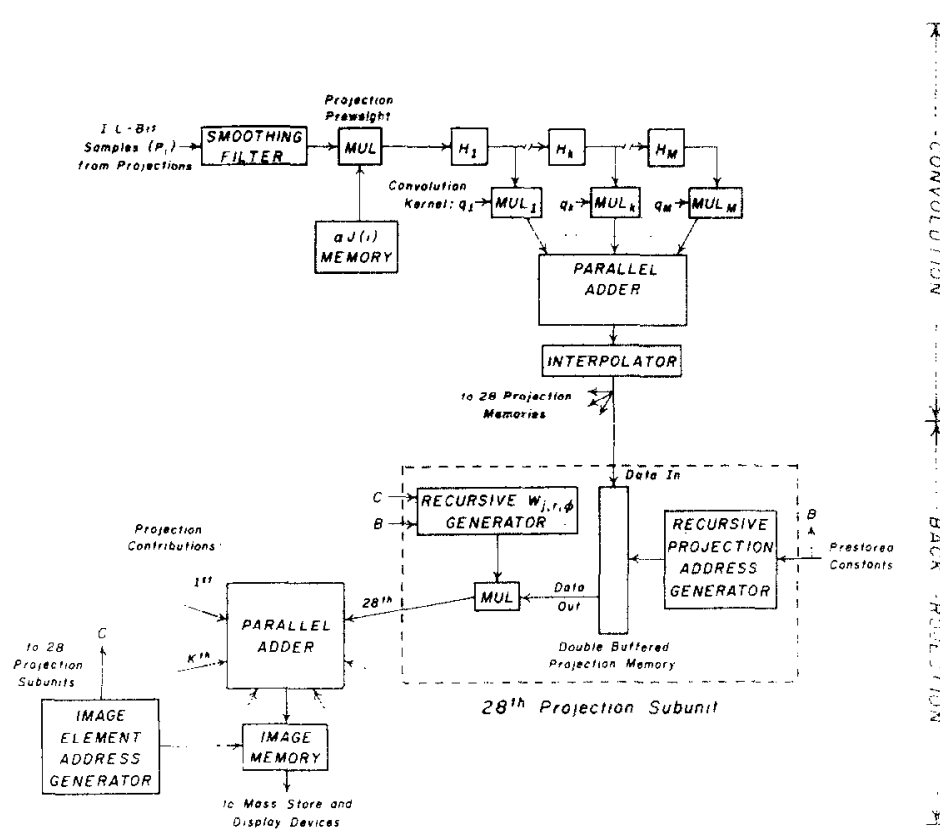

Fig. 10. Proposed architecture for a parallel processing hardwired reconstruction computer, in which look-up table memories of Fig. 2 are replaced by recursive generation of the $\hat{W}_{j, y, x}$ and ray sum indices via one or two stages of execution of Eq. [5]. In comparison to that of Fig. 2, this mechanization conserves memory hardware but decreases processor flexibility.

\section{SUMMARY}

The advent of a truly three-dimensional real-time X-ray transaxial tomography unit, the Dynamic Spatial Reconstructor, has been accompanied by a recognition of the magnitude of the task of processing the large volumes and bandwidths of digital data which are characteristic of DSR operation. It is demonstrated that reconstruction rates of 1 to $2 \mathrm{msec}$ per cross section can be achieved through the development of special purpose parallel processing computer achitectures capable of generating internal algorithmic constants via carefully formulated, computationally 
efficient numerical approximation techniques. Computer simulation studies indicate that no significant degradation of the final reconstructions should be expected, provided that the approximation methods implemented are sufficiently accurate.

\section{ACKNOWLEDGMENTS}

The authors aze indebted to Mr. Loren Krueger, Mr. Rodney Beistad, and Ms. M. T. Despres for technical assistance, and to Mr. Leo Johnson, Mrs. Delories Darling, Mrs. Pat Gustafson, Mrs. Donna Balow and co-workers in the Mayo Biodynamics Research Unit, for assistance in preparation of text and figures. This investigation was supported in part by U.S. Public Health Service Grants HL-14196, HL-04664, RR-00007 from the National Institutes of Health; National Aeronautics and Space Administration Grant NGR-24-003-001; U.S. Air Force Grant F49620-76-C-0001, National Science Foundation Grant MCS 77-03310, and grants from the Fannie E. Rippel Foundation and Control Data Corporation.

\section{REFERENCES}

1. Johnson, S. A. Total body exposure, pixel size, and signal-to-noise ratio considerations for threedimensional X-ray attenuation reconstructions: A convolutional derivation of reconstruction photon statistics. In "Reconstruction Tomography in Diagnostic Radiology and Nuclear Medicine" (M. M. Ter-Pogossian, M. E. Phelps, G. L. Brownell, J. R. Cox, D. O. Davis, and R. G. Evens, Eds.) pp. 199-200, University Park Press, Baltimore, 1977.

2. Inuma, T. A., Tateno, Y., Umegaki, Y., and Watanabe, E. Proposed system for ultrafast computed tomography. J. Comput. Assist. Tomography 1, 494-499 (1977).

3. Brooks, R. A. AND DiChIRo, G. Principles of computer-assisted tomography (CAT) in radiographic and radioisotopic imaging. Phys. Med. Biol. 21, 689-732 (1976).

4. WooD, E. H. New vistas for the study of structural and functional dynamics of the heart, lungs, and circulation by non-invasive numerical tomographic vivisection. Circulation 56, 506-520 (1977).

5. Sturm, R. E., Ritman, E. L., Johnson, S. A., Wondrow, M. A., Erdman, D. I., And Wood, E. H. Prototype of a single $\mathrm{X}$-ray video imaging chain designed for high temporal resolution computerized tomography by means of an electronic scanning dynamic spatial reconstruction system. In "Proceedings of the San Diego Biomedical Symposium" Vol. 15, pp. 181-188. Academic Press, New York, 1976.

6. Sturm, R. E., RTtMan, E. L., AND Wood, E. H. Prototype of a single X-ray video imaging chain designed for a fully electronic synchronous cylindrical scanning dynamic spatial reconstruction system. In: "Roentgen Video Techniques for Studies of Structure and Function of the Heart and Circulation" (P. H. Heintzen and J. H. Bursch, Eds.), pp. 261-270, Thieme, Stuttgart, 1978.

7. Ritman, E. L., Robb, R. A., Johnson, S. A., Chevalier, P. A., Gilbert, B. K., Greenleaf, J. F. STURm, R. E., AND Wood, E. H. Quantitative imaging of the structure and function of the heart, lungs, and circulation. Mayo Clin. Proc. 53, 3-11 (1978).

8. Ruegsegger, P. E., Ritman, E. L., AND Wood, E. H. Performance of an all electronic cylindrical CT scanning system for dynamic studies of the heart and lungs. In "Proceedings of the San Diego Biomedical Symposium 1977," Vol. 16, pp. 143-157. Academic Press, New York, 1977.

9. Robb, R. A., Johnson, S. A., Greenleaf, J. F., Wondrow, M. A., and Wood, E. H. An operator-interactive, computer-controlled system for high fidelity digitization and analysis of biomedical images. In "Proceedings of the Society of Photo-Optical Instrumentation Engineers 1973," Vol. 40, pp. 11-26, S.P.I.E. Press, Bellingham, WA. 
10. Ritman, E. L., Sturm, R. E., Robb, R. A., AND Wood, E. H. Needs, requirements, and design of a high temporal resolution synchronous cylindrical whole-body transaxial scanner for simultaneous study of the structure and function of the heart and circulation. In "Roentgen Video Techniques for Studies of Structure and Function of the Heart and Circulation" (P. H. Heintzen and J. H. Bursch, Eds.) pp. 271-284. Thieme, Stuttgart, Verlag, 1978.

11. Gilbert, B. K., Chu, A., And Rrtman, E. L. Data management, computation, and display techniyues for the dynamic spatial reconstruction of structural and functional information from the heart, lungs and circulatory system. In "Roentgen Video Techniques for Studies of Structure and Function of the Heart and Circulation" (P. H. Heintzen and J. H. Bursch, Eds.), pp. 300-312. Thieme, Stuttgart, 1978.

12. Gilbert, B. K., Beistad, R. D., Swartzlander, E. E., Jr., Krueger, L. M., Chu, A., Breuer, D. R. AND RITMAN, E. L. Development of very high-speed, multi-axial tomographic algorithms employing digital high-capacity fixed point arithmetic hardware. In "Proceedings of the San Diego Biomedical Symposium 1977," Vol. 16, pp. 159-168. Academic Press, New York, 1977.

13. Swartzlander, E. E., JR., Gilbert, B. K., and Reed. I. S. Inner product computers. IEEE Trans. Comput. C27, 21-31 (1978).

14. Reed, I. S., Kwoh, Y. S., Truong, T. K., and Hall. E. L. X-ray reconstruction by finite held transforms. IEEE Trans. Nucl. Sci. NS24, 843-849 (1977).

15. Herman, G. T., Lakshminarayanan, A. V., Naparstek, A., Ritman, E. L.. Robb, R. A., and Wood, E. H. Rapid computerized tomography. In "Proceedings of the Medical Data Processing Symposium 1976," Taylor \& Francis, London, pp. 581-598.

16. Swartzlander, E. E., Jr. Parallel counters. IEEE Trans. Comput. C22, 1021-1024 (I973).

17. Herman, G. T. and Naparsiek, A. Fast image reconstruction based on a Radon inversion formula appropriate for rapidly collected data. SIAM J. Appl. Math. 33, 511-533 (1977).

18. Herman, G. T., Hinds, J. A., Peretti, R. W., and Rowland, S. W. "A Programming System for the Reconstruction of Objects from Shadowgraphs." No. 94, Department of Computer Science, State University of New York, Buffalo, New York, 1975.

19. Sturm, R. E., Ritman, E. L. AND WOOD, E. H. Quantitative three-dimensional dynamic imaging of structure and function of the cardiopulmonary and circulatory systems in all regions of the body Cardiovas. Imag. and Image Proc., Theory and Practice 72, 103-122 (1976). 